\title{
O Uso do Método de Colocação na Resolução Equações Diferenciais Ordinárias
}

\author{
Daniela da Rosa Teza ${ }^{1}$ \\ Departamento Acadêmico de Matemática, UTFPR, Curitiba, PR, \\ Denise de Siqueira ${ }^{2}$ \\ Departamento Acadêmico de Matemática, UTFPR, Curitiba, PR
}

Resumo. O objetivo deste trabalho é aplicar o método da colocação para aproximar a solução de uma uma equação diferencial ordinária. No entanto os pontos de colocação adotados são os conhecidos "Nós de Chebyshev". Esta estratégia, também conhecida por Método Pseudo Espectral, permite entre outras coisas, evitar instabilidades da solução aproximada.

Palavras-chave. Método de Colocação, Equações Diferenciais Ordinárias, Polinômios Ortogonais.

\section{Introdução}

Resultados teóricos em muitos casos, nos garantem a existência e unicidade de soluções de equações diferenciais mas, não tem-se métodos analíticos para encontrar a solução e assim, o estudo de métodos numéricos de resolução se faz necessário. Em particular, utilizaremos os chamados "Métodos de Colocação" pois queremos aproximar a solução da equação diferencial ordinária por uma função definida num espaço de dimensão finita e, portanto, representada pelos elementos de sua base. O espaço sobre o qual procuraremos uma solução aproximada é o espaço de polinômios ortogonais, em particular o espaço de polinômios de Chebyshev.

\section{Desenvolvimento}

Os polinômios ortogonais têm como principal característica poderem ser gerados recursivamente e assim, se tornam computacionalmente atrativos. Além disso, sua ortogonalidade faz com que sistemas algébricos, sejam bem condicionados e com características que auxiliam na sua resolução. Por estes e outros motivos, utilizaremos os polinômios ortogonais como base para o espaço de aproximação no método de colocação.

\footnotetext{
${ }^{1}$ daniteza@gmail.com

${ }^{2}$ denisesiqueira@uftpr.edu.br
} 
A escolha dos pontos de colocação pode afetar drasticamente a estabilidade da solução aproximada e podemos nos deparar com o Fenômeno de Runge, que ocorre quando os pontos de colocação são igualmente espaçados. Porém, pode ser evitado fazendo uma escolha adequada desses pontos.

Considere o seguinte problema modelo: Encontrar $u$ tal que

$$
\begin{cases}u^{\prime \prime}(t) & =\sin (t) \\ u(1) & =u(-1)=0\end{cases}
$$

Como aproximação para a solução $u(t)$ da equação (1) tome $u_{h}=\sum_{j=0}^{N} \alpha_{j} \varphi_{j}(x)$, em que $\varphi_{j}(x)$ são os polinômios ortogonais, em particular, os polinômios de Chebyshev. Os pontos de colocação $t_{j}$ são os nós de Chebyshev, $t_{j}=\cos \left(\frac{\pi j}{N}\right), \operatorname{com} j=0, \ldots, N$.

A aproximação para a derivada, $u^{\prime \prime}(t)$ é dada por meio da matriz de diferenciação, segundo [3](página 53). Com essa estratégia, encontrar a solução de (1) é equivalente a resolver o sistema matricial $A \alpha=b$, onde $A$ é a matriz de diferenciação de ordem $(N+$ $1) \times(N+1), \alpha \in \mathbf{R}^{N+1}$ é o vetor de coeficientes e $b=\left(\sin \left(t_{j}\right)\right) \operatorname{com} j=0, \ldots, N$. A Figura 1 retrata a solução exata e aproximada do problema modelo. Por fim, podemos concluir que os polinômios ortogonais tornam o método adotado para aproximação, excelente.

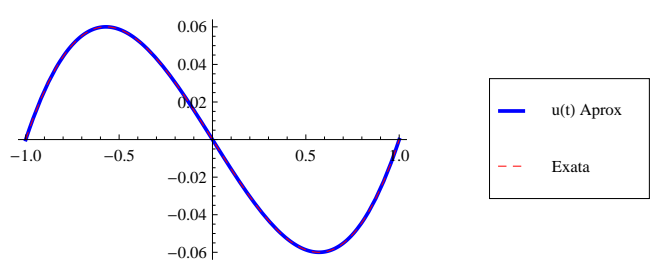

Figura 1: Soluções do problema modelo

\section{Agradecimentos}

Agradecemos à Universidade Tecnológica Federal do Paraná (UTFPR), ao Departamento Acadêmico de Matemática (DAMAT) e, ao Programa de Apoio a Pesquisa Científica e Desenvolvimento Tecnológico (PAPCDT).

\section{Referências}

[1] E. X. L. de Andrade,C. F. Bracciali e F. R. Rafaeli, Notas em matemática aplicada, SBMAC, vol. 64, (2012)

[2] R. L. Burden e J. D. Faires, Análise numérica, 8a. Edição, (2013)

[3] L. N. Trefethen, Spectral Methods in Matlab(Software, Environments, Tools), SIAM, (2000) 